\title{
Grain Iron, Zinc and Yield Genetics in Pearl Millet (Pennisetum glaucum L. R. Br.)
}

\author{
V.L. Ladumor ${ }^{1}$, K.D. Mungra ${ }^{2 *}$, S.K. Parmar ${ }^{2}$, J.S. Sorathiya ${ }^{2}$ and H.G. Vansjaliya ${ }^{2}$ \\ ${ }^{1}$ Department of Genetics and Plant Breeding, Junagadh Agricultural University, \\ Junagadh-362 001 Gujarat, India \\ ${ }^{2}$ Pearl Millet Research Station, Junagadh Agricultural University, Jamnagar-361 006, India \\ *Corresponding author
}

\section{A B S T R A C T}

\begin{tabular}{|l|}
\hline Key w or d s \\
Pearl millet \\
(Pennisetum \\
glaucum L. R. Br.)
\end{tabular}

The Line $\times$ Tester analysis involving 4 testers (females) and 7 lines (males) of pearl millet was carried out to identify crosses and good combiners for developing new hybrids to achieve high grain yield per plant. The variance due to GCA and SCA showed that the non-additive components were pre-dominant for the expression of days to flowering, days to maturity, plant height $(\mathrm{cm})$, number of effective tillers per plant (no.), dry fodder yield per plant (g), grain yield per plant (g) and Fe content (ppm) Whereas, additive components were predominant for the expression of ear head length $(\mathrm{cm})$, ear head diameter $(\mathrm{cm})$, dry fodder yield $(\mathrm{g})$, test weight $(\mathrm{g})$, harvest index and $\mathrm{Zn}$ content $(\mathrm{ppm})$. Among the female parents $\mathrm{ICMA}_{1} 10222$ and $\mathrm{JMSA}_{5} 20171$ were identified as good general combiner for grain yield per plant and some other component traits. Among the male parents 128-SB17, 160-SB-17 and 153-SB-17 were good general combiner for most of the characters. Among the 28 hybrids, two crosses $\left(\mathrm{JMSA}_{5} 20171 \times 153-\mathrm{SB}-17\right.$ and ICMA $12444 \times 118$ SB-17) were identified as good specific combiners based on significant and positive sca effect for grain yield per plant.

\section{Introduction}

Pearl millet [Pennisetum glaucum (L.) R. Br.] belongs to family Poaceae and genus Pennisetum. Pearl millet is the sixth most important and widely grown potential cereal crop in the world and is the fourth in India, after rice, wheat and maize. It is a highly cross-pollinated crop with protogynous flowering and wind borne pollination mechanism, which fulfill one of the essential biological requirements for hybrid development. Pearl millet is diploid $(2 n=14)$ in nature and commonly known as bajra, cat tail millet, and bulrush millet in different parts of the world, which is believed to be originated Africa. C4 species, it is endowed with a very high photosynthetic efficiency and more ability for dry matter production. Pearl millet is not only a quick growing short duration crop, but also found drought as well as heat tolerant and well adapted to different soil types. Because of its propensity for high dry matter production at high temperature, it has made a mark in tropics and sub-tropics. It is a drought resistant cereal having the 
maximum potentiality of grain production in adverse conditions. Pearl millet is an important coarse grain crop and serves as stable diet for the millions of people thriving under hunger. The better nutritive value of pearl millet grains appear from its protein, fat and mineral matters contents. It is also rich in vitamin $A$, vitamin $B$, thiamin as well as riboflavin contents and imparts substantial energy to the body with easy digestibility (Pal et al., 1996). Apart from grain, it also supplies fair quality of dry matter (forage and stover) at harvest in large bulk; which is an important secondary product in low resource agriculture for animal feed.

Combining ability studies are regarded useful to select best combining parents, which upon crossing would produce more desirable segregants. Such studies also elucidate the nature and magnitude of gene action involved in the inheritance of grain yield and its components, which will decide the breeding programme to be followed in segregating generations. They are several techniques for evaluating the varieties or lines in terms of their combining ability and genetic makeup. Among these, line $x$ tester analysis as proposed by Kempthorne (1957) has been extensively used to assess the combining ability of parent and crosses of different quantitative characters.

\section{Materials and Methods}

The experimental material for the present investigation comprised of four tester (female) obtained from ICRISAT and PMRS viz., $\mathrm{ICMA}_{1}$ 10222, ICMA 1 12444, JMSA 20155 , $\mathrm{JMSA}_{5} 20171$ and seven lines (males) developed at PMRS viz., 54-SB-17, 118-SB17, 127-SB-17, 128-SB-17, 130-SB-17, 153SB-17 and 160-SB-17. The material was obtained from Pearl Millet Research Station, Junagadh Agricultural University, Jamnagar. The checks included in this experiment were
GHB-732 and Dhanshakti. All the lines were crossed with four testers in Line $\times$ Tester $(\mathrm{L} \times$ $\mathrm{T})$ mating design to obtain 28 cross combinations. Evaluation of single cross hybrids, parents and checks were done in the Kharif, 2017. Five competitive plants from each experimental unit for every replication were selected randomly for recording observations on component characters viz., Days to flowering, days to maturity, plant height $(\mathrm{cm})$, number of effective tillers per plant (no.), ear head length $(\mathrm{cm})$, ear head diameter $(\mathrm{cm})$, test weight $(\mathrm{g})$, dry fodder yield per plant $(\mathrm{g})$, grain yield per plant $(\mathrm{g})$, harvest index, Fe content (ppm) and $\mathrm{Zn}$ content (ppm).

The combining ability analysis was carried out using line $\mathrm{x}$ tester mating design as per the procedure suggested by Kempthorn (1957).

\section{Results and Discussion}

The analysis of variance for combining ability for all the characters was carried-out according to the line $\times$ tester analysis proposed by Kempthorne (1957). The mean squares due to lines, tester and lines $\times$ tester were first tested against the error mean squares. If, line $\times$ tester interaction component found significant, the mean squares due to lines and testers were further tested against their respective interaction mean squares. The results obtained from the present study in respect to analysis of variance for combining ability (Table 1) are presented as under:

Partitioning of variances due to the crosses under investigation showed that the mean squares due to female (testers) were significant for ear head length, ear head diameter, test weight, dry fodder yield per plant and harvest index. Whereas, the mean squares due to male (lines) were found significant only for one character i.e. $\mathrm{Zn}$ content. The mean squares due to line $\times$ testers 
were found significant for days to flowering, days to maturity, plant height, dry fodder yield per plant, grain yield per plant and Fe content.

The mean squares due to female (testers) were found significant for all characters except dry fodder yield per plant when tested against mean square due to line $\times$ tester interaction. Similarly the mean squares due to male (lines) were also found significant for all the characters except plant height, and grain yield per plant when tested against mean square due to line $\times$ tester interaction.

The estimated variances due to female (tester) $\left(\sigma^{2} t\right)$ were higher than the corresponding variances due to male (lines) $\left(\sigma^{2} l\right)$ for all the characters except dry fodder yield per plant $\mathrm{Fe}$ content and $\mathrm{Zn}$ content.

The ratio of the mean squares components associated with variance of GCA and SCA was more than the unity for ear head length, ear head diameter, test weight, harvest index and $\mathrm{Zn}$ content indicating that the additive gene action was pre-dominant these characters. The ratio of ${ }^{2} \mathrm{GCA} /$ o $^{2} \mathrm{SCA}$ was less than the unity for days to flowering, days to maturity, plant height, number of effective tillers per plant, dry fodder yield per plant, grain yield per plant and $\mathrm{Fe}$ content these results tends to suggest that genetic variation among crosses was primarily of the nonadditive type. The results of the analysis of the variance for combing ability were also confirmed from the additive ( $\left.o^{2} \mathrm{~A}\right)$ and dominance ( $\left.o^{2} \mathrm{D}\right)$ components of variance.

The mean square due to line $x$ tester was significant revealing the non-additive type of gene action for the expression of days to flowering, days to maturity, plant height, number of effective tillers per plant and grain yield per plant. Similar results were also reported Bhadalia et al., (2014), Mungra et al., (2015) and Bagra, et al., (2017). The results of the present study indicated that the mean squares due to female (tester) was significant revealing the importance of additive type of gene action for the expression of ear head length, ear head diameter, test weight and harvest index in pearl millet. The predominance of additive gene action for these characters have been reported has been reported by Mungra et al., (2015) and Bagra, et al., (2017). The mean squares due to female (testers) and line $\times$ tester were significant revealing the importance of both additive and non-additive types of gene actions for the expression of dry fodder yield per plant. The predominance of non-additive gene action for dry fodder yield per plant has been reported by Yadav et al., (2012) and Bagra, et al., (2017). The mean squares due to line $\times$ tester were significant revealing the importance of nonadditive types of gene actions for the expression of Fe content.

The preponderance of non-additive gene action for $\mathrm{Fe}$ content has been reported by Arulselvi et al., (2009) and Kanatti et al., (2014). The significance of mean squares due to male (lines) suggested the additive type of gene actions for the expression of $\mathrm{Zn}$ content. The preponderance of additive gene action for $\mathrm{Zn}$ content has been reported by Jeeterwal et al., (2017).

The estimate of gca effects showed a wide range of variability among the parents in both the conditions (Table 2). None of the parents was good general combiner simultaneously for all the characters.

The females ICMA 10222 showed desirable gca effect and good general combiner for days to flowering, days to maturity, plant height, grain yield per plant and harvest index. Female ICMA $_{1} 12444$ was good general combiner for days to flowering, days to maturity, test weight, Fe content and $\mathrm{Zn}$ content. 
Table.1 Analysis of variance for combining ability and variance components for different characters in pearl millet

\begin{tabular}{|c|c|c|c|c|c|c|c|}
\hline Source & d. f. & $\begin{array}{l}\text { Days to } \\
\text { Flowering }\end{array}$ & Days to maturity & $\begin{array}{l}\text { Plant height } \\
(\mathrm{cm})\end{array}$ & $\begin{array}{l}\text { Number of effective } \\
\text { tillers per plant }\end{array}$ & $\begin{array}{c}\text { Ear head } \\
\text { length } \\
\text { (cm) }\end{array}$ & $\begin{array}{l}\text { Ear head diameter } \\
\qquad(\mathrm{cm})\end{array}$ \\
\hline & & 1 & 2 & 3 & 4 & 5 & 6 \\
\hline Replications & 2 & 0.58 & 2.39 & 1759.91 & 0.06 & $20.97 * *$ & 0.19 \\
\hline Tester (Female) & 3 & $100.07+$ & $160.87+$ & $751.75+$ & $0.03+$ & $13.93 *+$ & $0.91 * *++$ \\
\hline Lines (male) & 6 & $51.60+$ & $68.25+$ & 193.03 & $0.03+$ & $4.57+$ & $0.29+$ \\
\hline Line $\times$ Tester & 18 & $50.01 * *$ & $60.78 * *$ & $370.83^{*}$ & 0.02 & 3.85 & 0.12 \\
\hline Error & 54 & 0.29 & 0.48 & 182.81 & 0.08 & 3.12 & 0.07 \\
\hline \multicolumn{8}{|c|}{ Variance components } \\
\hline$\sigma^{2} t$ (female) & - & 4.75 & 7.64 & 27.09 & -0.00 & $0.52 *$ & $0.04 * *$ \\
\hline$\sigma^{2} 1$ (male) & - & 4.28 & 5.65 & 0.85 & -0.00 & 0.12 & 0.02 \\
\hline$\sigma^{2} \mathrm{lt}$ & - & $16.57 * *$ & $20.10 * *$ & $62.67 * *$ & 0.02 & 0.24 & 0.02 \\
\hline$\sigma^{2}$ gca & - & $4.58 *$ & $6.91 *$ & 17.55 & -0.00 & $0.38 * *$ & $0.03 * *$ \\
\hline$\sigma^{2} \operatorname{sea}$ & - & $16.57 * *$ & $20.10 * *$ & $62.67 * *$ & 0.016 & 0.24 & 0.02 \\
\hline$\sigma^{2}$ gca / $\sigma^{2}$ sca & - & 0.28 & 0.34 & 0.28 & -0.18 & 1.53 & 1.77 \\
\hline \multirow[t]{2}{*}{ Source } & d. f. & $\begin{array}{l}\text { Test weight } \\
\text { (g) }\end{array}$ & $\begin{array}{l}\text { Dry fodder yield } \\
\text { per plant (g) }\end{array}$ & $\begin{array}{c}\text { Grain yield per } \\
\text { plant (g) }\end{array}$ & $\begin{array}{c}\text { Harvest index } \\
(\%)\end{array}$ & $\begin{array}{l}\text { Fe content } \\
(\text { ppm })\end{array}$ & $\begin{array}{l}\text { Zn content } \\
(\mathbf{p p m})\end{array}$ \\
\hline & & 8 & 9 & 10 & 11 & 12 & 13 \\
\hline Replications & 2 & 2.54 & 20.93 & 46.89 & 14.03 & 25.76 & 105.86 \\
\hline Female (Testers) & 3 & $14.99 * *++$ & $55.43 *$ & $173.59+$ & $249.88 * *++$ & $334.75+$ & $111.98+$ \\
\hline Male (Lines) & 6 & $5.53+$ & $155.99+$ & 44.65 & $40.17+$ & $369.66+$ & $153.16^{*}+$ \\
\hline Line $\times$ Tester & 18 & 2.89 & $93.05^{*}$ & $57.23 * *$ & 25.14 & $316.99 * *$ & 49.81 \\
\hline Error & 54 & 1.62 & 49.94 & 24.63 & 16.54 & 100.60 & 49.69 \\
\hline \multicolumn{8}{|c|}{ Variance components } \\
\hline$\sigma^{2} t$ (female) & - & $0.63 * *$ & 0.26 & 7.093 & $11.11 * *$ & 11.15 & 2.966 \\
\hline$\sigma^{2} 1$ (male) & - & 0.33 & 8.84 & 1.66 & 1.97 & 22.42 & $8.62 *$ \\
\hline$\sigma^{2}$ lt & - & 0.42 & $14.37 *$ & $10.87 * *$ & 2.87 & $72.13 * *$ & 0.04 \\
\hline$\sigma^{2}$ gca & - & $0.52 * *$ & 3.38 & 5.12 & $7.79 * *$ & 15.25 & 5.02 \\
\hline$\sigma^{2}$ sca & - & 0.42 & $14.37 *$ & $10.87 * *$ & 2.87 & $72.13 * *$ & 0.04 \\
\hline$\sigma^{2}$ gea / $\sigma^{2}$ sca & - & 1.23 & 0.23 & 0.47 & 2.72 & 0.21 & 129.12 \\
\hline
\end{tabular}

$*$, ** Significant at 5 and 1 per cent levels, respectively when tested against error mean square.

,+++ Significant at 5 and 1 per cent levels, respectively when tested against lines $\mathrm{x}$ testers interaction mean square. 
Table.2 General combining ability effects of parents for different characters in pearl millet

\begin{tabular}{|c|c|c|c|c|c|c|c|c|c|c|c|c|c|}
\hline \multirow[t]{3}{*}{$\begin{array}{l}\text { Sr. } \\
\text { No. }\end{array}$} & Parents & $\begin{array}{l}\text { Days to } \\
\text { flowering }\end{array}$ & $\begin{array}{l}\text { Days to } \\
\text { maturity }\end{array}$ & $\begin{array}{l}\text { Plant } \\
\text { height } \\
\text { (cm) }\end{array}$ & $\begin{array}{c}\text { Number of } \\
\text { effective } \\
\text { tillers of } \\
\text { plant }\end{array}$ & $\begin{array}{c}\text { Ear } \\
\text { head } \\
\text { length } \\
(\mathrm{cm})\end{array}$ & $\begin{array}{c}\text { Ear } \\
\text { head } \\
\text { diameter } \\
(\mathbf{c m})\end{array}$ & $\begin{array}{c}\text { Test } \\
\text { weight } \\
\text { (g) }\end{array}$ & $\begin{array}{c}\text { Dry } \\
\text { fodder } \\
\text { yield per } \\
\text { plant (g) }\end{array}$ & $\begin{array}{l}\text { Grain } \\
\text { yield per } \\
\text { plant (g) }\end{array}$ & $\begin{array}{c}\text { Harvest } \\
\text { index } \\
(\%)\end{array}$ & $\begin{array}{l}\text { Fe content } \\
\quad(\mathbf{p p m})\end{array}$ & $\begin{array}{c}\mathbf{Z n} \\
\text { conten } \\
\mathbf{t} \\
(\mathrm{ppm})\end{array}$ \\
\hline & & 1 & 2 & 3 & 4 & 5 & 6 & 7 & 8 & 9 & 10 & 11 & 12 \\
\hline & \multicolumn{13}{|c|}{ Testers (Female) } \\
\hline 1 & $\begin{array}{l}\text { ICMA }_{1} \\
10222\end{array}$ & $\begin{array}{r}-1.99 * * \\
(\mathrm{G})\end{array}$ & $\begin{array}{r}-2.42 * * \\
(\mathrm{G})\end{array}$ & $\begin{array}{r}7.501 * \\
(\mathrm{G})\end{array}$ & $0.03(\mathrm{~A})$ & $-0.57(\mathrm{~A})$ & $\begin{array}{r}-0.11 * \\
(\mathrm{P})\end{array}$ & $0.45(\mathrm{~A})$ & $1.22(\mathrm{~A})$ & $\begin{array}{r}2.21 * \\
(\mathrm{G})\end{array}$ & $\begin{array}{r}2.32 * * \\
(\mathrm{G})\end{array}$ & $2.07(\mathrm{~A})$ & $\begin{array}{r}-0.63 \\
(\mathrm{~A})\end{array}$ \\
\hline 2 & $\begin{array}{l}\text { ICMA }_{1} \\
12444\end{array}$ & $\begin{array}{r}-1.37 * * \\
(\mathrm{G})\end{array}$ & $\begin{array}{r}-1.75^{* *} \\
(\mathrm{G})\end{array}$ & $0.64(\mathrm{~A})$ & $-0.06(\mathrm{~A})$ & $-0.65(\mathrm{~A})$ & $\begin{array}{r}-0.23 * * \\
\text { (P) }\end{array}$ & $\begin{array}{r}0.59 * \\
(\mathrm{G})\end{array}$ & $-2.01(\mathrm{~A})$ & $-1.65(\mathrm{~A})$ & $0.31(\mathrm{~A})$ & $4.59 *(\mathrm{G})$ & $\begin{array}{r}3.27 * \\
(\mathrm{G})\end{array}$ \\
\hline 3 & $\begin{array}{l}\mathrm{JMSA}_{5} \\
20155\end{array}$ & $0.49(\mathrm{~A})$ & $0.44(\mathrm{~A})$ & $\begin{array}{r}-7.03 * * \\
(\mathrm{P})\end{array}$ & $-0.01(\mathrm{~A})$ & $0.11(\mathrm{~A})$ & $\begin{array}{r}0.12^{*} \\
(\mathrm{G})\end{array}$ & $\begin{array}{r}-1.25 * * \\
(\mathrm{P})\end{array}$ & $1.41(\mathrm{~A})$ & $\begin{array}{r}-3.19 * * \\
(\mathrm{P})\end{array}$ & $\begin{array}{r}-4.97 * \\
(\mathrm{P})\end{array}$ & $-3.02(\mathrm{~A})$ & $\begin{array}{r}-0.49 \\
\text { (A) }\end{array}$ \\
\hline \multirow[t]{4}{*}{4} & $\begin{array}{l}\mathrm{JMSA}_{5} \\
20171\end{array}$ & $\begin{array}{r}2.87 * * \\
(\mathrm{P})\end{array}$ & $\begin{array}{r}3.73 * * \\
(\mathrm{P})\end{array}$ & $-1.12(\mathrm{~A})$ & $-0.03(\mathrm{~A})$ & $\begin{array}{r}1.11 * * \\
(\mathrm{G})\end{array}$ & $\begin{array}{r}0.23 * * \\
(\mathrm{G})\end{array}$ & $0.22(\mathrm{~A})$ & $-0.63(\mathrm{~A})$ & $\begin{array}{r}2.64 * \\
(G)\end{array}$ & $\begin{array}{r}2.34 * * \\
(\mathrm{G})\end{array}$ & $-3.64(\mathrm{~A})$ & $\begin{array}{r}-2.15 \\
(\mathrm{~A})\end{array}$ \\
\hline & $\operatorname{SE}\left(\mathbf{g}_{\mathbf{i}}\right)$ & 0.12 & 0.15 & 2.95 & 0.06 & 0.38 & 0.05 & 0.28 & 1.54 & 1.08 & 0.89 & 2.19 & 1.54 \\
\hline & $\operatorname{SE}\left(g_{i-} g_{j}\right)$ & 0.16 & 0.21 & 4.17 & 0.08 & 0.54 & 0.08 & 0.39 & 2.18 & 1.53 & 1.25 & 3.09 & 2.17 \\
\hline & \multicolumn{13}{|c|}{ Lines (Male) } \\
\hline 1 & 54-SB-17 & $\begin{array}{r}2.39 * * \\
(\mathrm{P})\end{array}$ & $\begin{array}{r}2.55 * * \\
(\mathrm{P})\end{array}$ & $5.44(\mathrm{~A})$ & $0.01(\mathrm{~A})$ & $-0.73(\mathrm{~A})$ & $0.03(\mathrm{~A})$ & $0.59(\mathrm{~A})$ & $-2.90(\mathrm{~A})$ & $-0.74(\mathrm{~A})$ & $1.25(\mathrm{~A})$ & $1.73(\mathrm{~A})$ & $\begin{array}{r}-1.04 \\
(\mathrm{~A})\end{array}$ \\
\hline 2 & $\begin{array}{l}118-S B- \\
17\end{array}$ & $\begin{array}{r}-1.36 * * \\
(\mathrm{G})\end{array}$ & $\begin{array}{r}-1.70 * * \\
(\mathrm{G})\end{array}$ & $-4.67(\mathrm{~A})$ & $-0.04(\mathrm{~A})$ & $0.06(\mathrm{~A})$ & $\begin{array}{r}-0.14 * \\
(\mathrm{P})\end{array}$ & $\begin{array}{r}-1.09 * * \\
(\mathrm{P})\end{array}$ & $-3.86(\mathrm{~A})$ & $\begin{array}{r}-0.64 \\
(\mathrm{~A})\end{array}$ & $\begin{array}{r}2.30 * \\
(\mathrm{G})\end{array}$ & $\begin{array}{r}-11.61 * * \\
(\mathrm{P})\end{array}$ & $\begin{array}{r}-6.95 \\
(\mathrm{~A})\end{array}$ \\
\hline 3 & $\begin{array}{l}\text { 127-SB- } \\
17\end{array}$ & $\begin{array}{r}1.73 * * \\
(\mathrm{P})\end{array}$ & $\begin{array}{r}2.29 * * \\
(\mathrm{P})\end{array}$ & $1.09(\mathrm{~A})$ & $-0.02(\mathrm{~A})$ & $-0.48(\mathrm{~A})$ & $0.02(\mathrm{~A})$ & $0.21(\mathrm{~A})$ & $2.65(\mathrm{~A})$ & $2.52(\mathrm{~A})$ & 0.97 (A) & $0.98(\mathrm{~A})$ & $\begin{array}{r}4.96^{*} \\
(\mathrm{G})\end{array}$ \\
\hline 4 & $\begin{array}{l}128-S B- \\
17\end{array}$ & $2.31 * *(\mathrm{P})$ & $\begin{array}{r}2.63 * * \\
(\mathrm{P})\end{array}$ & $0.74(\mathrm{~A})$ & 0.06 (A) & $0.29(\mathrm{~A})$ & $\begin{array}{r}0.19 * \\
(\mathrm{G})\end{array}$ & $0.01(\mathrm{~A})$ & $3.69(\mathrm{~A})$ & $2.98 *(\mathrm{G})$ & 0.41 (A) & $5.48(\mathrm{~A})$ & $\begin{array}{r}0.71 \\
(\mathrm{~A})\end{array}$ \\
\hline 5 & $\begin{array}{l}130-S B- \\
17\end{array}$ & $-0.77 *(\mathrm{G})$ & $\begin{array}{r}-0.95 * \\
(G)\end{array}$ & $-1.11(\mathrm{~A})$ & $-0.07(\mathrm{~A})$ & $0.14(\mathrm{~A})$ & $\begin{array}{r}-0.27 * * \\
(\mathrm{P})\end{array}$ & $-0.65(\mathrm{~A})$ & $-1.75(\mathrm{~A})$ & $-1.68(\mathrm{~A})$ & $-0.99(\mathrm{~A})$ & $2.23(\mathrm{~A})$ & $\begin{array}{r}0.88 \\
(\mathrm{~A})\end{array}$ \\
\hline 6 & $\begin{array}{l}153-S B- \\
17\end{array}$ & $\begin{array}{c}-2.36 * * \\
(\mathrm{G})\end{array}$ & $\begin{array}{r}-2.45 * * \\
(\mathrm{G})\end{array}$ & $-5.27(\mathrm{~A})$ & $0.01(\mathrm{~A})$ & $-0.40(\mathrm{~A})$ & 0.09 (A) & $0.86^{*}(\mathrm{G})$ & $-2.69(\mathrm{~A})$ & $-1.62(\mathrm{~A})$ & $-0.68(\mathrm{~A})$ & 2.89 (A) & $\begin{array}{r}0.38 \\
(\mathrm{~A})\end{array}$ \\
\hline 7 & $\begin{array}{l}160-S B- \\
17\end{array}$ & $\begin{array}{c}-1.94 * * \\
\text { (G) }\end{array}$ & $\begin{array}{r}-2.37 * * \\
(\mathrm{G})\end{array}$ & $3.78(\mathrm{~A})$ & $0.03(\mathrm{~A})$ & $1.11 *(\mathrm{G})$ & $0.07(\mathrm{~A})$ & $0.08(\mathrm{~A})$ & $4.86^{*}(\mathrm{G})$ & $-0.81(\mathrm{~A})$ & $\begin{array}{r}-3.26 * * \\
(\mathrm{P})\end{array}$ & $-1.69(\mathrm{~A})$ & $\begin{array}{r}1.05 \\
(\mathrm{~A})\end{array}$ \\
\hline & $\operatorname{SE}\left(\mathbf{g}_{\mathbf{j}}\right)$ & 0.15 & 0.19 & 3.90 & 0.08 & 0.51 & 0.08 & 0.37 & 2.04 & 1.43 & 1.17 & 2.89 & 2.03 \\
\hline & $\operatorname{SE}\left(\mathbf{g}_{\left.\mathbf{i}-g_{\mathbf{j}}\right)}\right.$ & 0.22 & 0.28 & 5.52 & 0.11 & 0.72 & 0.11 & 0.52 & 2.88 & 2.05 & 1.66 & 4.09 & 2.88 \\
\hline
\end{tabular}


Table.3 Specific combining ability effects of crosses for different characters in pearl millet

\begin{tabular}{|c|c|c|c|c|c|c|c|}
\hline \multirow[t]{2}{*}{$\begin{array}{l}\text { Sr. } \\
\text { No. }\end{array}$} & \multirow[t]{2}{*}{ Crosses } & $\begin{array}{l}\text { Days to } \\
\text { flowering }\end{array}$ & $\begin{array}{l}\text { Days to } \\
\text { maturity }\end{array}$ & $\begin{array}{c}\text { Plant height } \\
\text { (cm) }\end{array}$ & $\begin{array}{c}\text { Number of } \\
\text { effective tillers per } \\
\text { plant }\end{array}$ & $\begin{array}{l}\text { Ear head } \\
\text { length } \\
\text { (cm) }\end{array}$ & $\begin{array}{l}\text { Ear head } \\
\text { diameter } \\
\quad(\mathrm{cm})\end{array}$ \\
\hline & & 1 & 2 & 3 & 4 & 5 & 6 \\
\hline 1 & $\mathrm{ICMA}_{1} 10222 \times 54-\mathrm{SB}-17$ & $-2.68 * *$ & $-2.83 * *$ & -5.42 & 0.22 & 0.17 & -0.05 \\
\hline 2 & $\mathrm{ICMA}_{1} 10222 \times 118-\mathrm{SB}-17$ & $1.07 * *$ & $1.08 * *$ & 7.89 & 0.01 & 0.94 & $0.25 *$ \\
\hline 3 & $\mathrm{ICMA}_{1} 10222 \times 127-\mathrm{SB}-17$ & $2.32 * *$ & $3.08 * *$ & -0.94 & 0.12 & -0.53 & 0.11 \\
\hline 4 & $\mathrm{ICMA}_{1} 10222 \times 128-\mathrm{SB}-17$ & $-3.59 * *$ & $-4.25 * *$ & 0.01 & -0.09 & 0.07 & $-0.24 *$ \\
\hline 5 & $\mathrm{ICMA}_{1} 10222 \times 130-\mathrm{SB}-17$ & $1.49 * *$ & $2.00 * *$ & -9.87 & -0.16 & 0.49 & $0.24 *$ \\
\hline 6 & $\mathrm{ICMA}_{1} 10222 \times 153-\mathrm{SB}-17$ & 0.40 & 0.17 & 10.43 & -0.04 & 0.04 & -0.18 \\
\hline 7 & $\mathrm{ICMA}_{1} 10222 \times 160-\mathrm{SB}-17$ & $0.99 * *$ & 0.75 & -2.09 & -0.06 & $-1.18 *$ & -0.13 \\
\hline 8 & $\mathrm{ICMA}_{1} 12444$ x 54-SB-17 & $-3.96 * *$ & $-4.17 * *$ & -3.49 & -0.09 & -0.15 & 0.21 \\
\hline 9 & $\mathrm{ICMA}_{1} 12444$ x 118-SB-17 & $-0.88 * *$ & $-1.25 * *$ & 7.16 & $0.29 *$ & -0.69 & -0.18 \\
\hline 10 & $\mathrm{ICMA}_{1} 12444$ x $127-\mathrm{SB}-17$ & $-3.96 * *$ & $-4.58 * *$ & -7.94 & 0.14 & 0.33 & -0.12 \\
\hline 11 & $\mathrm{ICMA}_{1} 12444$ x $128-\mathrm{SB}-17$ & $9.79 * *$ & $10.75 * *$ & -0.59 & -0.21 & $1.38 *$ & $0.32 *$ \\
\hline 12 & $\mathrm{ICMA}_{1} 12444$ x $130-\mathrm{SB}-17$ & $1.20 * *$ & $1.67 * *$ & -4.34 & -0.21 & -0.39 & -0.03 \\
\hline 13 & $\mathrm{ICMA}_{1} 12444$ x $153-\mathrm{SB}-17$ & $-2.21 * *$ & $-2.50 * *$ & 9.69 & 0.11 & 0.58 & -0.22 \\
\hline 14 & $\mathrm{ICMA}_{1} 12444$ x 160-SB-17 & 0.04 & 0.08 & -0.49 & -0.04 & -1.07 & 0.01 \\
\hline 15 & $\mathrm{JMSA}_{5} 20155$ x 54-SB-17 & $8.84 * *$ & $9.31 * *$ & $18.24 *$ & -0.02 & $0.99 *$ & 0.05 \\
\hline 16 & $\mathrm{JMSA}_{5} 20155$ x $118-\mathrm{SB}-17$ & $-0.74 *$ & -0.77 & -12.84 & 0.03 & -1.14 & -0.14 \\
\hline 17 & $\mathrm{JMSA}_{5} 20155 \times 127-\mathrm{SB}-17$ & $-1.15 * *$ & $-1.11 * *$ & 8.73 & -0.19 & $0.99 *$ & -0.04 \\
\hline 18 & $\mathrm{JMSA}_{5} 20155 \times 128-\mathrm{SB}-17$ & $-3.74 * *$ & $-3.77 * *$ & -0.86 & 0.13 & $-1.94 * *$ & 0.04 \\
\hline 19 & $\mathrm{JMSA}_{5} 20155 \times 130-\mathrm{SB}-17$ & $-3.32 * *$ & $-4.19 * *$ & 13.73 & 0.26 & 0.54 & -0.01 \\
\hline 20 & $\mathrm{JMSA}_{5} 20155 \times 153-\mathrm{SB}-17$ & $2.26 * *$ & $2.98 * *$ & $-27.64 * *$ & $-0.29 *$ & $-1.28 *$ & 0.19 \\
\hline 21 & $\mathrm{JMSA}_{5} 20155$ x 160-SB-17 & $-2.15 * *$ & $-2.44 * *$ & 0.64 & 0.09 & $1.84 * *$ & -0.08 \\
\hline 22 & $\mathrm{JMSA}_{5} 20171$ x 54-SB-17 & $-2.20 * *$ & $-2.31 * *$ & -9.33 & -0.11 & $-1.01 *$ & -0.21 \\
\hline 23 & $\mathrm{JMSA}_{5} 20171$ x 118-SB-17 & 0.55 & $0.94 *$ & -2.21 & $-0.33 *$ & 0.89 & 0.06 \\
\hline 24 & $\mathrm{JMSA}_{5} 20171 \times 127-\mathrm{SB}-17$ & $2.79 * *$ & $2.61 * *$ & 0.15 & -0.08 & -0.79 & 0.05 \\
\hline 25 & $\mathrm{JMSA}_{5} 20171$ x 128-SB-17 & $-2.45 * *$ & $-2.73 * *$ & 1.44 & 0.17 & 0.49 & -0.11 \\
\hline 26 & $\mathrm{JMSA}_{5} 20171 \times 130-\mathrm{SB}-17$ & $0.63 *$ & 0.52 & 0.49 & 0.11 & -0.65 & -0.20 \\
\hline 27 & $\mathrm{JMSA}_{5} 20171 \times 153-\mathrm{SB}-17$ & -0.45 & -0.64 & 7.52 & 0.22 & 0.66 & 0.21 \\
\hline 28 & $\mathrm{JMSA}_{5} 20171 \times 160-\mathrm{SB}-17$ & $1.13 * *$ & $1.61 * *$ & 1.94 & 0.01 & 0.41 & 0.20 \\
\hline & $\mathbf{S E}\left(\mathbf{S}_{\mathrm{ij}}\right)$ & 0.31 & 0.39 & 7.81 & 0.16 & 1.02 & 0.15 \\
\hline & $\mathbf{S E}\left(\mathbf{S}_{\mathrm{ij}}-\mathbf{S}_{\mathrm{kl}}\right)$ & 0.44 & 0.56 & 11.04 & 0.25 & 1.44 & 0.21 \\
\hline
\end{tabular}

*, ** Significant at $5 \%$ and $1 \%$ levels, respectively 
Cont...

\begin{tabular}{|c|c|c|c|c|c|c|c|}
\hline \multirow[t]{2}{*}{$\begin{array}{l}\text { Sr. } \\
\text { No. }\end{array}$} & \multirow[t]{2}{*}{ Crosses } & $\begin{array}{c}\text { Test weight } \\
\text { (g) }\end{array}$ & $\begin{array}{l}\text { Dry fodder yield } \\
\text { per plant } \\
\text { (g) }\end{array}$ & $\begin{array}{c}\text { Grain yield per } \\
\text { plant } \\
\text { (g) }\end{array}$ & $\begin{array}{c}\text { Harvest index } \\
(\%)\end{array}$ & $\begin{array}{c}\text { Fe content } \\
(\text { ppm })\end{array}$ & $\begin{array}{l}\text { Zn content } \\
\text { (ppm) }\end{array}$ \\
\hline & & 7 & 8 & 9 & 10 & 11 & 12 \\
\hline 1 & $\mathrm{ICMA}_{1} 10222 \times 54-\mathrm{SB}-17$ & -1.12 & $-7.54 *$ & -3.22 & 1.21 & -1.82 & 1.46 \\
\hline 2 & ICMA $_{1} 10222$ x $118-S B-17$ & 0.62 & 1.81 & 1.57 & 0.80 & -6.82 & -2.62 \\
\hline 3 & ICMA $_{1} 10222$ x $127-$ SB-17 & -1.13 & $7.18^{*}$ & 4.24 & -0.84 & -8.74 & -5.54 \\
\hline 4 & $\mathrm{ICMA}_{1} 10222 \times 128-\mathrm{SB}-17$ & 0.55 & 3.36 & 0.62 & -1.27 & -0.90 & 0.71 \\
\hline 5 & $\mathrm{ICMA}_{1} 10222 \times 130-\mathrm{SB}-17$ & $1.74 *$ & -1.19 & 2.17 & 1.57 & $14.68^{*}$ & $6.88 *$ \\
\hline 6 & $\mathrm{ICMA}_{1} 10222 \times 153-\mathrm{SB}-17$ & -0.55 & -4.13 & -3.22 & -0.49 & 2.34 & 1.38 \\
\hline 7 & $\mathrm{ICMA}_{1} 10222 \times 160-\mathrm{SB}-17$ & -0.11 & 0.53 & -2.15 & -0.97 & 1.26 & -2.29 \\
\hline 8 & $\mathrm{ICMA}_{1} 12444$ x 54-SB-17 & 0.35 & 3.19 & -0.25 & -3.44 & $-13.34 *$ & -5.44 \\
\hline 9 & $\mathrm{ICMA}_{1} 12444$ x $118-\mathrm{SB}-17$ & 0.05 & 3.48 & $6.87 *$ & 3.38 & -2.01 & -0.19 \\
\hline 10 & $\mathrm{ICMA}_{1} 12444$ x $127-\mathrm{SB}-17$ & 0.63 & 2.30 & -0.51 & -0.50 & 5.07 & 1.56 \\
\hline 11 & $\mathrm{ICMA}_{1} 12444$ x $128-\mathrm{SB}-17$ & 0.62 & -3.41 & -1.97 & 0.01 & 8.24 & $3.14 *$ \\
\hline 12 & ICMA $_{1} 12444$ x 130-SB-17 & $-1.79 *$ & -6.41 & -5.14 & -0.79 & 4.15 & 0.98 \\
\hline 13 & ICMA $_{1} 12444$ x $153-$ SB-17 & 0.44 & 3.63 & -0.70 & -1.88 & -1.18 & -1.52 \\
\hline 14 & ICMA $_{1} 12444$ x 160-SB-17 & -0.31 & -2.78 & 1.70 & 3.22 & -0.93 & 1.48 \\
\hline 15 & $\mathrm{JMSA}_{5} 20155$ x 54-SB-17 & $1.46^{*}$ & $7.55^{*}$ & -1.04 & -3.69 & $21.61 * *$ & $4.99 *$ \\
\hline 16 & $\mathrm{JMSA}_{5} 20155$ x 118-SB-17 & 0.03 & -3.05 & -3.80 & -1.32 & 3.27 & 1.23 \\
\hline 17 & $\mathrm{JMSA}_{5} 20155$ x 127-SB-17 & -0.31 & -4.63 & 2.70 & $4.41 *$ & -2.31 & 1.99 \\
\hline 18 & $\mathrm{JMSA}_{5} 20155$ x $128-\mathrm{SB}-17$ & -0.34 & 2.62 & 3.06 & -0.32 & -0.14 & -2.09 \\
\hline 19 & $\mathrm{JMSA}_{5} 20155$ x 130-SB-17 & 0.13 & 4.17 & 3.02 & 1.38 & -6.56 & -1.59 \\
\hline 20 & $\mathrm{JMSA}_{5} 20155 \times 153-\mathrm{SB}-17$ & -0.70 & $-8.21 *$ & -4.19 & 1.73 & -1.23 & 0.90 \\
\hline 21 & $\mathrm{JMSA}_{5} 20155 \times 160-\mathrm{SB}-17$ & -0.28 & 1.55 & 0.25 & -2.18 & $-14.64 *$ & -5.43 \\
\hline 22 & $\mathrm{JMSA}_{5} 20171$ x 54-SB-17 & -0.69 & -3.19 & 4.51 & $5.92 *$ & -6.44 & -1.01 \\
\hline 23 & $\mathrm{JMSA}_{5} 20171 \times 118-\mathrm{SB}-17$ & -0.70 & -2.24 & -4.64 & -2.86 & 5.56 & 1.57 \\
\hline 24 & $\mathrm{JMSA}_{5} 20171 \times 127-\mathrm{SB}-17$ & 0.80 & -4.86 & $-6.43^{*}$ & -3.07 & 5.98 & 1.99 \\
\hline 25 & $\mathrm{JMSA}_{5} 20171 \times 128-\mathrm{SB}-17$ & -0.83 & -2.57 & -1.71 & 1.57 & -7.19 & -1.76 \\
\hline 26 & $\mathrm{JMSA}_{5} 20171 \times 130-\mathrm{SB}-17$ & -0.08 & 3.43 & -0.04 & -2.15 & $-12.27 *$ & -6.26 \\
\hline 27 & $\mathrm{JMSA}_{5} 20171 \times 153-\mathrm{SB}-17$ & 0.81 & $8.71 *$ & $8.12 *$ & 0.64 & 0.06 & -0.76 \\
\hline 28 & $\mathrm{JMSA}_{5} 20171 \times 160-\mathrm{SB}-17$ & 0.69 & 0.71 & 0.19 & -0.06 & $14.31 *$ & $6.24 *$ \\
\hline & $\mathbf{S E}\left(\mathbf{S}_{\mathrm{ij}}\right)$ & 0.73 & 4.08 & 2.86 & 2.35 & 5.79 & 4.07 \\
\hline & $\operatorname{SE}\left(\mathbf{S}_{\mathrm{ij}}-\mathbf{S}_{\mathrm{kl}}\right)$ & 1.04 & 5.77 & 4.05 & 3.32 & 8.19 & 5.76 \\
\hline
\end{tabular}

*, ** Significant at $5 \%$ and $1 \%$ levels, respectively 
Female $\mathrm{JMSA}_{5} 20155$ was good general combiner for ear head diameter and female JMSA $_{5} 20171$ was good general combiner for ear head length, ear head diameter, grain yield per plant and harvest index. The male parents 118-SB-17, 130-SB-17, 153-SB-17 and 160SB-17 were good general combiner for days to flowering and days to maturity. The male parent 160-SB-17 was good general combiner for ear head length and dry fodder yield per plant. The male parent 128-SB-17 was good combiner for ear head diameter and grain yield per plant. The parent 153-SB-17 was good general combiner for test weight. The parent 118-SB-17 was good general combiner for harvest index. The parent 127-SB-17 was good general combiner for $\mathrm{Zn}$ content (Table $3)$.

The estimates of sca effects revealed that none of the crosses had simultaneously significant for all the characters. The 12 and 14 crosses exhibited significant negative sca effect for days to flowering and days to maturity respectively. The highest desirable negative sca effect was exhibited by the cross $\mathrm{ICMA}_{1} 12444 \times 127-\mathrm{SB}-17$ followed by $\mathrm{ICMA}_{1} 12444 \times 54-\mathrm{SB}-17, \mathrm{JMSA}_{5} 20155 \times$ 128-SB-17, ICMA $10222 \times 128-$ SB-17 and $\mathrm{ICMA}_{1} 20155 \times 130-\mathrm{SB}-17$. For plant height, only one cross $\mathrm{JMSA}_{5} 201555 \times 54-\mathrm{SB}-17$ showed significant and positive sca effect. Only one cross ICMA $_{1} 12444 \times 118-$ SB- 17 showed significant sca effect in desirable direction for number of effective tillers per plant. Three crosses showed significant and positive sca effect for ear head length. The highest positive effect was exhibited by the cross $\mathrm{JMSA}_{5} 20155 \times 160-\mathrm{SB}-17$ followed by $\mathrm{ICMA}_{1} 12444 \times 128-\mathrm{SB}-17$ and JMSA 20155 $\times$ 54-SB-17. Three crosses as good specific combiners as they showed significant and positive sca effect for ear head diameter. The maximum sca effect was displayed by the cross $\mathrm{ICMA}_{1} 12444 \times 128-\mathrm{SB}-17$ followed by $\mathrm{ICMA}_{1} 10222 \times 118-\mathrm{SB}-17$ and $\mathrm{ICMA}_{1}$
$12444 \times 130-\mathrm{SB}-17$. The crosses $\mathrm{ICMA}_{1}$ $10222 \times 130-$ SB-17 and JMSA $520155 \times 54-$ SB-17 showed significant positive sca effect for test weight. Three crosses showed significant positive sca effect for dry fodder yield per plant. Out of these, the maximum significant and positive sca effect was exhibited by the cross $\mathrm{JMSA}_{5} 20171 \times 153-$ SB-17 followed by JMSA $20155 \times 54-S B-17$ and $\mathrm{ICMA}_{1} 10222 \times 127-\mathrm{SB}-17$. For grain yield per plant, the crosses $\mathrm{JMSA}_{5} 20171 \times$ 153-SB-17 and ICMA $12444 \times 118-$ SB-17 were identified as good specific combiners based on significant and positive sca effect. The crosses, JMSA $520171 \times 54-S B-17$ and $\mathrm{JMSA}_{5} 20155 \times 127-\mathrm{SB}-17$ were showed significant and positive sca effect for harvest index. The 3 and 4 crosses showed significant and positive sca effect for Fe content and $\mathrm{Zn}$ content respectively. The highest positive and significant sca effect was exhibited by the cross $\mathrm{JMSA}_{5} 20155 \times 54-\mathrm{SB}-17$ followed by $\mathrm{ICMA}_{1} 10222 \times 130-\mathrm{SB}-17$ and $\mathrm{JMSA}_{5} 20171$ $\times 160-$ SB-17.

From the present findings it can be concluded that sufficient variation was present in the material for grain yield and its components. Both additive and non-additive genetic variances were found important in the expression of all the traits. The additive gene action was more important for the five characters such as ear head length, ear head diameter, test weight, harvest index and $\mathrm{Zn}$ content. Thus, it would be possible to improve these traits through pedigree breeding method. The preponderance of non- additive genetic variance was observed in the inheritance for seven characters such as days to flowering, days to maturity, plant height, dry fodder yield per plant, grain yield per plant and $\mathrm{Fe}$ content. This suggested that heterosis breeding or bi-parental mating would be more suitable for the improvement of these traits in pearl millet. The female $\mathrm{ICMA}_{1} 10222$ and $\mathrm{JMSA}_{5} 20171$ and the 
male 128-SB-17 displayed high gca effect and for grain yield per plant and some desirable traits like plant height, ear head length, ear head diameter and harvest index. Therefore, these parents were identified as good general combiners and could be preferred in breeding programme as these parents upon crossing, are expected to give desirable segregants in the succeeding generations.

The crosses $\mathrm{JMSA}_{5} 20171 \times 153-\mathrm{SB}-17$ and and $\mathrm{ICMA}_{1} 12444 \times 118-\mathrm{SB}-17$ displayed high sca effect for grain yield per plant. The high sca status of the hybrids indicated that substantial role was also played by dominance and epistatic interaction. Such crosses could be exploited through heterosis breeding.

\section{References}

Arulselvi, S., Mohanasundaram, K. and Selvi, B. 2009. Genetic analysis of grain quality characters and grain yield in pearl millet [Pennisetum glaucum (L.) R. Br.]. Crop Res., 37(1/3): 161-167.

Bagra, S.K., Mungra, K.D. and Sorathiya, J.S. 2017. Grain yield and blast disease genetic architecture in pearl millet (Pennisetum glaucum (L.) R. Br.). AGRES- An Int. e-J., 6(1): 147-155.

Bhadalia, A.S., Dhedhi, K.K., Joshi, H.J. and Sorathiya, J.S. 2014. Combining ability studies through diallel analysis in pearl millet [Pennisetum glaucum (L.) R. Br.]. Int. J. Agri. Sci., 10(1): 57-60.

Jeeterwal, R. C., Sharma, L. D. and Nehra, A. 2017. Combining ability and heterosis for grain iron and zinc content in pearl millet [Pennisetum glaucum (L.)]. Int. J. Chem. Std., 5(4): 472-475.

Kanatti, A., Rai, K.N., Radhika, K. and Govindaraj, M. 2016. Tester Effect on Combining Ability and Its Relationship with Line Performance per se for Grain Iron and Zinc Densities in Pearl Millet. Crop Sci., 56(2): 689-696.

Kempthorne, O. 1957. An Introduction to Genetic Statistics. John Willey \& Sons. Inc., New York.

Mungra, K.S., Dobariya, K.L., Sapovadiya, M.H. and Vavadiya, P.A. 2015. Combining ability and gene action for grain yield and its component tarits in pearl millet (Pennisetum glaucum (L.) R. Br.). Elect. J. pl. Breed., 6(1): 66-73.

Pal, M., Daka, J. and Rai, R. K. 1996. Fundamentals of Cereal Crop Production. Tata Mc Grow Hill Publishing Company Limited, New Delhi.

Yadav, A.K., Nirmalakumari, A. and Arya, R.K. 2012. Study of genetic architecture for maturity traits in relation to supraoptimal temperature tolerance in pearl millet (Pennisetum glaucum (L.) R. Br.). Int. J. Pl. Bred. Genet., 6: 115-128.

\section{How to cite this article:}

Ladumor, V.L., K.D. Mungra, S.K. Parmar, J.S. Sorathiya and Vansjaliya, H.G. 2018. Grain Iron, Zinc and Yield Genetics in Pearl Millet (Pennisetum glaucum L. R. Br.). Int.J.Curr.Microbiol.App.Sci. 7(09): 242-250. doi: https://doi.org/10.20546/ijcmas.2018.709.031 